\title{
Strong (Weak) Domination Intuitionistic Fuzzy Graphs
}

\author{
J. John Stephan \\ Assistant professor, \\ Department of Mathematics \\ Dhanalakshmi srinivasan \\ Engineering college, \\ Perambalur.
}

\author{
A. Muthaiyan \\ Assistant professor \\ P.G \& Research department of \\ Mathematics, \\ Government Arts College. \\ Ariyalur
}

\author{
N. Vinoth Kumar \\ Assistant professor, \\ Department of Mathematics, \\ M.A.M. School of Engineering, \\ Trichy
}

\begin{abstract}
Let $\mathrm{G}$ be an IFG. Then $D \subseteq V$ is said to be a strong (weak) dominating set if every $v \in V-D$ is strongly (weakly) dominated by some vertex in $\mathrm{D}$. We denote the strong (weak) intunionistic fuzzy dominating set by sid-set (wid-set).The minimum vertex cardinality over all the sid-set (wid-set) is called the strong (weak) dominating number of an IFG and is denoted by $\gamma_{\text {sid }}(G)\left[\gamma_{\text {wid }}(G)\right]$.In this paper, introduce the strong (weak) domination in intunionistic fuzzy graphs and obtain some bounds in IFG.
\end{abstract}

\section{Keywords}

Intuitionistic fuzzy graph, strong (weak) domination, strong (weak) domination number

\section{INTRODUCTION}

The first definition of fuzzy graphs was proposed by Kafmann, from the fuzzy relations introduced by Zadeh. Although Rosenfeld introduced another elaborated definition, including fuzzy vertex and fuzzy edges, and several fuzzy analogs of graph theoretic concepts such as paths, cycles, connectedness and etc. The concept of domination in fuzzy graphs was investigated by A. Somasundaram, S. Somasundaram and A. Somasundaram present the concepts of independent domination, total domination, connected domination of fuzzy graphs [11] . C. Natarajan and S.K. Ayyaswamy introduce the strong (weak) domination in fuzzy graph [13]. The first definition of intuitionistic fuzzy graphs was proposed by Atanassov. [3] The concept of domination in intuitionistic fuzzy graphs was investigated by R.parvathi and G.Thamizhendhi. In this paper we develop the strong (weak) domination set of intuitionistic fuzzy graphs. Further introduce a dominating parameter of the sets and investigate the property of this domination parameter in intuitionistic fuzzy graph.

\section{BASIC DEFINITIONS}

An intuitionistic fuzzy graph (IFG) is of the form $\mathrm{G}=(\mathrm{V}, \mathrm{E})$, where $\quad V=\left\{v_{1}, v_{2}, \ldots, v_{n}\right\}$ such that $\mu_{1}: V \rightarrow[0,1], \gamma_{1}: V \rightarrow[0,1]$ denote the degree of membership and nonmember ship of the element $v_{i} \in V$ respectively and $0 \leq \mu_{1}\left(v_{i}\right)+\gamma_{1}\left(v_{i}\right) \leq 1$ for every $v_{i} \in V,(i=1,2, \ldots n) . E \subseteq V \times V$ Where $\mu_{2}: V \times V \rightarrow[0,1]$, and $\gamma_{2}: V \times V \rightarrow[0,1]$ are such that

$$
\mu_{2}\left(v_{i}, v_{j}\right) \leq \mu_{1}\left(v_{i}\right) \wedge \mu_{1}\left(v_{j}\right),
$$

$\gamma_{2}\left(v_{i}, v_{j}\right) \leq \gamma_{1}\left(v_{i}\right) \vee \gamma_{1}\left(v_{j}\right)$ and

$$
\begin{aligned}
& 0 \leq \mu_{2}\left(v_{i}, v_{j}\right)+\gamma_{2}\left(v_{i}, v_{j}\right) \leq 1 \text {. } \\
& \text { An } \operatorname{arc}\left(v_{i}, v_{j}\right) \text { of an IFG } \mathrm{G} \text { is called } \\
& \text { an strong arc if } \\
& \mu_{2}\left(v_{i}, v_{j}\right)=\mu_{1}\left(v_{i}\right) \wedge \mu_{1}\left(v_{j}\right), \\
& \gamma_{2}\left(v_{i}, v_{j}\right)=\gamma_{1}\left(v_{i}\right) \wedge \gamma_{1}\left(v_{j}\right) \text {. } \\
& \text { Let } \mathrm{G}=(\mathrm{V}, \mathrm{E}) \text { be an IFG. Then the } \\
& \text { cardinality of } \mathrm{G} \text { is defined to be } \\
& |G|=\left|\sum_{v_{i} \in V}\left[\frac{\left(1+\mu_{1}\left(v_{i}\right)-\gamma_{1}\left(v_{i}\right)\right)}{2}\right]\right|+\left|\sum_{v_{i} \in V}\left[\frac{\left(1+\mu_{2}\left(v_{i}, v_{j}\right)-\gamma_{1}\left(v_{i}, v_{j}\right)\right)}{2}\right]\right| \\
& \text { Let } \mathrm{G}=(\mathrm{V}, \mathrm{E}) \text { be an IFG. The vertex } \\
& \text { cardinality of } \mathrm{G} \text { is defined to be } \\
& |G|=\left|\sum_{v_{i} \in V}\left[\frac{\left(1+\mu_{1}\left(v_{i}\right)-\gamma_{1}\left(v_{i}\right)\right)}{2}\right]\right| \quad \text { for } \quad \text { all } \\
& v_{i} \in V,(i=1,2, \ldots n) \\
& \text { Let } \mathrm{G}=(\mathrm{V}, \mathrm{E}) \mathrm{b} \text { e an IFG. An edge cardinality } \\
& \text { of } \mathrm{G} \text { is defined to be } \\
& |G|=\left|\sum_{v_{i} \in V}\left[\frac{\left(1+\mu_{2}\left(v_{i}, v_{j}\right)-\gamma_{1}\left(v_{i}, v_{j}\right)\right)}{2}\right]\right| \quad \text { for } \quad \text { all } \\
& \left(v_{i}, v_{j}\right) \in V \times V \text {, }
\end{aligned}
$$

Let $\mathrm{G}=(\mathrm{V}, \mathrm{E})$ be an IFG. A set $D \subseteq V$ is said to be a dominating set o G if every $v \in V-D$ there exist $u \in D$ such that $\mathrm{u}$ dominates $\mathrm{v}$.

An intuionistic fuzzy dominating D of an IFG, G is called minimal dominating set of $\mathrm{G}$ if every node $u \in D$, $D-\{u\}$ is not a dominating set in G.

An intunionistic fuzzy domination number $\gamma_{i f}(G)$ of an IFG, $G$ is the minimum vertex cardinality over all minimal dominating sets in $\mathrm{G}$.

A set $S \subseteq V$ in an IFG, G is said to be an independent if there is no strong between the vertices $v \in S$. An independent set $S$ of IFG , G is said to be maximal independent set if every node $v \in V-S$ then the set $S \cup\{v\}$ is not an independent set in G. The minimum cardinality among all the maximal independent sets in an IFG, $\mathrm{G}$ is called the intunionistic fuzzy independent number.. 


\section{STRONG (WEAK) VERTEX DOMINATION}

\subsection{Neighbor of a Node}

Let $\mathrm{G}$ be an IFG. The neighbor of a node $v \in V$ is defined by $N(v)=\left\{\begin{array}{r}u \in v: \mu_{2}(u v)=\mu_{1}(u) \wedge \mu_{1}(v) \\ \gamma_{2}(u v)=\gamma_{1}(u) \vee \gamma_{1}(v)\end{array}\right\}$

\subsection{Neighborhood Degree of a Node}

The vertex cardinality of $\mathrm{N}(\mathrm{v})$ is the neighborhood degree of $\mathrm{v}$ which is denoted by $d_{N}(v)$. $d_{N}(v)=\sum_{u \in N(v)}\left[\frac{1+\mu_{1}(u)-\gamma_{1}(u)}{2}\right]$

\subsection{Effective degree of a node}

The effective degree of $\mathrm{v}$ is an edge cardinality of the strong edge incident v. $d_{E}(v)=\sum_{u \in V}\left[\frac{1+\mu_{2}(u v)-\gamma_{2}(u v)}{2}\right] \mathrm{uv}$ is a strong arc

\subsection{Strong (weak) Vertex Domination}

Let $\mathrm{u}$ and $\mathrm{v}$ be any two vertex in an IFG, G. Then $\mathrm{u}$ strongly dominates $\mathrm{v}$ ( $\mathrm{v}$ weakly dominates $\mathrm{u}$ ) if (i). $\mathrm{uv}$ is a strong arc (ii) $d_{N}(u) \geq d_{N}(v)$.

\section{Theorem 3.4.1}

Let $\mathrm{D}$ be a minimal strong dominating set of an IFG,G. then for each $v \in D$. One of the following holds

1) No vertex in D strongly dominates $\mathrm{v}$

2) There exist $u \in V-D$ such that $\mathrm{v}$ is the only vertex in $\mathrm{D}$ which strongly dominates $\mathrm{u}$.

Proof:

Let $\mathrm{D}$ be a minimal strong dominating set of an IFG, G. any vertex $v \in D$, then clearly $\mathrm{D}-\{\mathrm{v}\}$ is not a strong dominating set in G, by the minimal property. Therefore we note that no vertex in $\mathrm{D}$ strongly dominates $\mathrm{v}$.

Let $u \in V-D, \mathrm{D}$ is a minimal strong dominating set. Assume $v, w \in D$ strongly dominates $u \in V-D$, Then D$\mathrm{w}$ is a strong dominating set. Therefore $\mathrm{D}$ is not a minimal strong dominating set of $\mathrm{G}$. this is contradiction to our assumption. Therefore we get $u \in V-D$ such that $\mathrm{v}$ is the only vertex in $\mathrm{D}$ which strongly dominates $\mathrm{v}$.

\section{Theorem 3.4.2}

For an IFG, G $(\mathrm{V}, \mathrm{E})$ of order P

$$
\begin{aligned}
& \gamma_{i f}(G) \leq \gamma_{s d}(G) \leq p-\Delta_{N}(G) \leq p-\Delta_{E}(G) \\
& \gamma_{i f}(G) \leq \gamma_{w d}(G) \leq p-\delta_{N}(G) \leq p-\delta_{E}(G)
\end{aligned}
$$

Proof:

Every strong (weak) dominating set is a dominating

$$
\begin{aligned}
& \text { set of an IFG, G. } \quad \gamma_{i f}(G) \leq \gamma_{s d}(G) \text {, } \\
& \gamma_{i f}(G) \leq \gamma_{w d}(G) .
\end{aligned}
$$

Let $u, v \in V$. If $d_{N}(u)=\Delta_{N}(G), d_{N}(v)=\delta_{N}(G)$. Clearly we get $V-N(u)$ is a strong dominating set and $V-N(v)$ is a weak dominating set of $\mathrm{G}$. Therefore $\gamma_{s d}(G) \leq|V-N(u)|_{i f}=p-\Delta_{N}(G)$ $\gamma_{w d}(G) \leq|V-N(v)|_{i f}=p-\delta_{N}(G)$.

Further since $\Delta_{E}(G) \leq \Delta_{N}(G)$ and $\delta_{E}(G) \leq \delta_{N}(G)$ therefore we get $\gamma_{i f}(G) \leq \gamma_{s d}(G) \leq p-\Delta_{N}(G) \leq p-\Delta_{E}(G)$ $\gamma_{i f}(G) \leq \gamma_{w d}(G) \leq p-\delta_{N}(G) \leq p-\delta_{E}(G)$.

\section{Theorem 3.4.3}

If $\mathrm{D}$ is a minimal strong (weak) dominating set of a connected IFG G, then V-D is weak (strong) dominating set of G.

Proof:

Assume D is a minimal dominating set of an IFG, G . Let $u \in V-D$, there exist a vertex $v \in D$ such that (i). uv is a strong $\operatorname{arc}$ (ii) $d_{N}(v) \geq d_{N}(u)$. Therefore we note u weakly dominates $\mathrm{v}$ in $\mathrm{G}$. Clearly $V-D$ is a weak dominating set of $\mathrm{G}$.

Similarly we prove for minimal weak domination sets.

\section{Theorem 3.4.4}

For a complete IFG, G. $v, u \in V$ be the vertices having the minimum and maximum vertex cardinality in $\mathrm{G}$ respectively.

$$
\begin{aligned}
& \gamma_{s d}(G)=\left[\frac{1+\mu_{1}(v)-\gamma_{1}(v)}{2}\right] \text { and } \\
& \gamma_{w d}(G)=\left[\frac{1+\mu_{1}(u)-\gamma_{1}(u)}{2}\right]
\end{aligned}
$$

Proof:

Let $\mathrm{G}$ be a complete IFG. There exists an edge between every pair of vertices. So we get degree of an every vertices is of the form $d_{N}(v)=\sum_{u \in V-u} d_{N}(u)$. Clearly we get if $\mathrm{v}$ is having a minimal vertex cardinality $d_{N}(v) \geq d_{N}(u)$, for every $u \in V-v$. Therefore we get

$\{v\}$ is a minimal strong dominating set of $G$

$$
\gamma_{s d}(G)=\left[\frac{1+\mu_{1}(v)-\gamma_{1}(v)}{2}\right]
$$

Similarly we prove $\gamma_{w d}(G)=\left[\frac{1+\mu_{1}(u)-\gamma_{1}(u)}{2}\right]$. 


\section{Example}

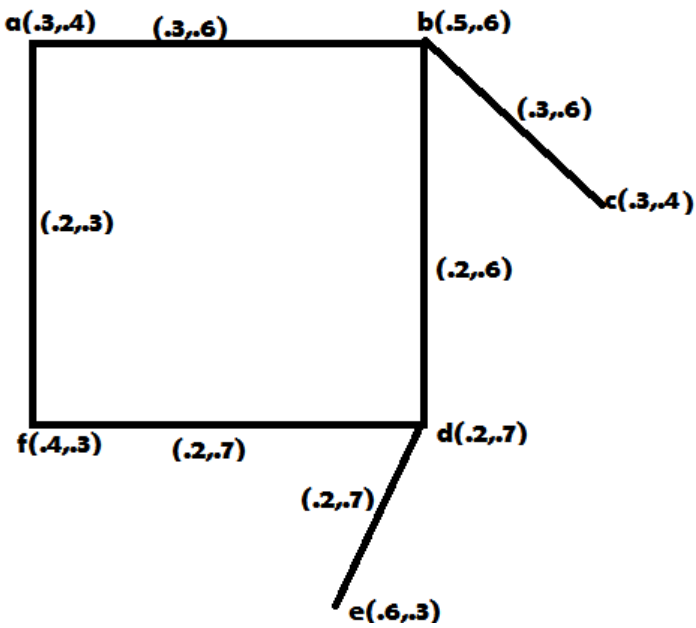

For the above fuzzy graphs, SD set $D=\{b, d\}$ and

$\gamma_{s d}(G)=.65$

\section{APPLICATION}

Let $G$ be a graph which represents the railway network of a state. Let the vertices denote the junctions and the edges denote the roads connecting the junctions. The membership functions, non membership functions $\mu_{1}, \mu_{2}, \gamma_{1}$ and $\gamma_{2}$ on the vertex set and the edge set of $G$ can be constructed from the statistical data that represents the number of trains passing through various junctions and the number of trains passing through various roads during a time interval. Thus we get an intutionistic fuzzy graph $G$. In this IFG a vertex edge fuzzy dominating set $D$ can be interpreted as the set of junctions in which trains crossing is heavier than the other junctions not in D.

\section{CONCLUSION}

The concept of constant IFG in graphs is very rich both in theoretical developments and applications. In this paper, we introduced strong domination set and number some interesting properties of these new concepts are proved.

\section{REFERENCES}

[1] Arumugam.S and Velammal.S, Edge Domination in Graphs, Taiwanese Journal Of Mathematics, Vol.2, No.2, pp.173-179, June 1998

[2] Ayyaswamy.S, and Natarajan.C, International Journal of Computational and Mathematical sciences, 2010.

[3] K. Atanassov, Intuitionistic Fuzzy Sets: Theory and Applications, Physica-Verlag, New York (1999).

[4] Harary.F., Graph Theory, Addition Wesely, Third Printing, October 1972.

[5] Mordeson,J.N., and Nair, P.S., Fuzzy graphs andFuzzy Hyper graphs, Physica-Verlag, Heidelberg,1998,second edition, 2001.

[6] Nagoor Gani, A., and Vadivel.P, Relation between the Parameter of Independent Domination and Irredundance in Fuzzy Graph, International Journal of Algorithms, Computing and Mathematics, Vol 2,2009. of Algorithms, Computing and Mathematics, Vol 2,2009.

[7] Nagoor Gani A, Shajithab Begum S, Degree, Order and Size in Intuitionistic Fuzzy Graphs, International journal of algorithms, Computing and Mathematics, Volume 3, Number 3, August 2010

[8] Somasundaram,A.,Somasundaram,S.,1998,domination in Fuzzy Graphs-I, Pattern Recognition Letters, 19, pp. 787-791.

[9] Somasundaram, A., 2004, Domination in Fuzzy Graph-II, Journal of Fuzzy Mathematics, 20

[10] R.B.Allan and R.Laskar, On domination and independant domination of a graph, Discrete Math. 234 (1978), 73 76

[11] Somasundaram, A., Somasundaram, S., 1998 Domination in Fuzzy Graphs-I, Pattern

[12] Nagoor Gani, A, and Basher Ahamed.M, Order and Size in Fuzzy Graphs, Bulletin of Pure and Applied Science, Vol 22E, 2003; p.145-148

[13] C.Natarajan and S.K.Ayyaswamy, on strong weak domination in fuzzy graphs, World Academy of Science, Engineering and Technology, Vol:4 2010-07-24 